\title{
CARACTERIZACIÓN DEL ABUSO SEXUAL INFANTIL A PARTIR DE HISTORIAS CLÍNICAS
}

\section{Characterization of child sexual abuse from medical records}

\author{
Leslie Bravo García y Yira Meléndez Monroy* \\ Corporación Universitaria del Caribe, CECAR. Colombia
}

\begin{abstract}
Resumen
El abuso sexual es una problemática que afecta a niños y niñas en todos los contextos y en especial Colombia. Registrando un alto índice de menores abusados sexualmente, por ello la presente investigación realiza una categorización de este flagelo. Como metodología, se utilizó el análisis de historias clínicas, con un instrumento validado por expertos, en los que se estableció que ambos sexos están propensos al abuso, siendo ligeramente superior en las niñas que en los niños, en cuanto al contexto donde mayormente se presenta esta problemática es en la familia, y el tipo principal de abuso que se exterioriza es con contacto físico; de igual manera, se resalta el hecho que la mayoría de los eventos abusivos se muestran en las zonas urbanas.
\end{abstract}

Palabras Claves: Caracterización, abuso sexual infantil, historias clínicas.

\begin{abstract}
The problem of sexual abuse affects children in all contexts and especially Colombia. Registering a high rate of sexually abused children, so this research provides a categorization of this scourge. As methodology, analysis of medical records, with a validated by experts instrument, in which it was established that both sexes are prone to abuse, being slightly higher in girls than in boys, as the context in which mostly occurs was used this problem is in the family, and the main type of abuse that is physical contact externalized; similarly, it highlights the fact that most abusive events are shown in urban areas.
\end{abstract}

Keywords: Characterization, child sexual abuse, medical records.

\footnotetext{
* Corporación Universitaria del Caribe. CECAR .Colombia. leslie.bravo@cecar.edu.co
} 


\section{INTRODUCCIÓN}

$\mathrm{El}$ abuso sexual se considera un signo de maltrato que atenta con las esferas físicas y psicologías del niño, niña o adolescente, vulnerando sus derechos en su integridad y dignidad humana, a tal punto de originar consecuencias a corto, a mediano y largo plazo (Alonso y Horno, 2004) Según (Losada, 2012) en los casos de abuso sexual infantil entre 1946 y 2011 a nivel mundial el $18,95 \%$ se da en mujeres y el $9,7 \%$ en varones. Siendo esta cifra alarmante puesto que involucra a la infancia y adolescencia en pleno desarrollo de sus capacidades físicas y psicológicas.

Este flagelo que ataca a la población infantil es una problemática de índole universal, presente en todas las culturas, lo cual no es fácil determinar que tanto se ha incrementado en comparación con otras épocas, como consecuencia de la desunión familiar, del interés de los medios de comunicación y profesionales, señalando que para poder precisarlo hace falta realizar estudios comparativos. Lo que sí es real, es que hoy día se conocen más, aunque siguen algunos casos que no salen a luz (Echeburua y Corral, 2006).

Colombia no es ajena a esta situación, dado que en el reporte de medicina legal de 2013 se conocieron 1.333 casos de violencia sexual a menores de 17 años, siendo los menores de 14 años los más afectados, estas cifras corresponden a los meses de Enero a Septiembre del 2013. El 83\% afecta a las niñas, considerando que cada hora dos niñas son abusadas sexualmente y los abusadores de mayor índice son los realizados por un familiar, seguido por conocido, desconocido, amigo y vecino. De acuerdo a datos estadísticos el Instituto Colombiano de Bienestar Familiar (ICBF, 2013) reporto que entre enero y agosto, 4.710 niños, niñas y adolescentes han sufrido de algún tipo de violencia sexual, los cuales se les ha brindado protección e intervención terapéutica. Además se estima que de cada ocho infantes que llegan a ICBF uno de ellos ha sido violentado sexualmente. La mayoría está bajo la amparo del ICBF, dado que los padres no ofrecen garantías para su cuidado y otros fueron dados en adopción.

En el Departamento de Sucre, según los directivos del centro de atención a víctimas del abuso sexual en Sincelejo (CAVAS, 2014) indicaron que no hay una cultura denunciante en cuanto a casos de Así. Esto por temor al escarnio público de la familia, dificultando así la labor de intervención de los organismos responsables de llevar a cabo campañas de atención y prevención a la población afectada. Afectación que conlleva en algunos casos a una experiencia traumática ocasionando heridas en el aparato psíquico, alterando y confundiendo al niño, niña o adolescente en su estructura mental.

Siguiendo con los estudios en cuanto a este tema Pereda (2011) en una revisión que hace de artículos internacionales relacionados con víctimas de abuso sexual en la infancia y los trastornos de personalidad, encontró en mayor número de frecuencia, el trastorno antisocial y límite, señalando que "los trabajos son pocos concluyentes" sugiriendo la importancia de realizar investigaciones longitudinales. Por su parte Mebarak (2010) habla acerca de la sintomatología del abuso sexual infantil, y determinó que la experiencia deja diferentes secuelas de tipo psicopatológico surgiendo la necesidad de seguir investigando el tema, dado que existen contradicciones que no logran clarificar con exactitud la sintomatología.

Debido a la sintomatología y consecuencias del abuso sexual que anteriormente se ha mencionado, se ha activado una alerta en el reconocimiento de tipo social y profesional del abuso sexual, notándose un incremento en el número de casos conocidos aunque probablemente ello representa únicamente una pequeña parte de la incidencia real (Rodríguez, 2003. Por tal motivo y de acuerdo a los reportes e información que se recoge en cuanto a esta problemática, se hace necesario en la presente investigación caracterizar el abuso sexual en niños y niñas de 6-14 años de edad describiendo los factores personales, contexto, sexo, edad y escolaridad, tanto de la víctima como el victimario, para luego analizar los indicadores físicos y psicológicos más frecuentes de los abusos sexuales a través de las historias clínicas registrados en un centro de atención a víctimas de abuso sexual Sucre 2014. El abuso sexual es una temática de investigación que torna difícil para trabajar dado que posee componentes como lo es el secreto, la vergüenza y la culpa, de esta forma se pone en evidencia en evidencia distintos aspectos controvertidos de la sociedad hoy, como los pueden ser la sexualidad, el poder, los valores y actitudes hacia la infancia, 
El abuso sexual es una conducta de tipo sexual con un niño o adolescente llevada a cabo por un adulto o por otro similar a él. Generalmente el abuso sexual se realiza tomando al menor a la fuerza, engañándolo, comprándolo, perturbándolo emocionalmente o haciendo valer la autoridad o poder que se tiene sobre él. El abusador conquista la confianza del niño o niña, ganando su amor y afecto y por lo cual él o ella accede. El abusador antes de irrumpir el cuerpo, atacó la psiquis, dominando su intimidad. Este se gana al niño haciéndole creer que él lo permitió y es culpable, culpabilidad que ayuda a instaurar el secreto manteniendo el silencio que posiblemente nunca es revelado, dejando consecuencias a largo plazo en la adultez (Losada, 2009).

Los delincuentes sexuales o pedófilos satisfacen su sexualidad por medio de cualquier tocamiento, penetración o solo gratificarse con el solo hecho de verlo desnudo involucrando otras acciones de este tipo en donde no hay tocamiento y que no sea necesario el contacto físico con el cuerpo del niño o niña, pero que si repercuten en la su salud mental de estos de la misma forma que sucede con las víctimas de abuso sexual con contacto. Ante este hecho puede hablarse de un abuso sexual con contacto y sin contacto. (Horno, Santos y Del Molino, 2001).

Por otra parte en lo concerniente a la víctima, el vivir o haber experimentado ser abusado repercute de forma negativa en su desarrollo psicosexual, afectivo, social y moral, y a su vez las consecuencias del abuso en algunos individuos pueden mantenerse en el tiempo y reactivarse en el curso de la vida de la persona abusada. Existen, algunas características distintivas del abuso sexual que también permite clasificarlos como Características Del Contexto: Donde ocurre es decir a nivel intrafamiliar o extra familiar. En este sentido, las investigaciones han mostrado que los casos de abuso sexual más frecuentes son realizados por personas cercanas al niño; y en especial a nivel intrafamiliar (Intebi, 1998), ante esto la persona abusadora puede llegar a ser el padre, la madre, abuelo o abuela, un tío o tía, el hermano o hermana, algún primo o prima, y por ende padrastro o madrastra en familias de tipo resconstituida.

Además conviene subrayar que Faller 1993 (citado por Manso, 2006) en el modelo integrador relaciona las diferencias entre las condiciones y los factores que favorecen el hecho de vulnerabilidad de la víctima, pero no lo provocan. Dentro de las Condiciones se encuentran: Factores relacionados al sistema social vigente, como educación específica para cada sexo, reparto rígido de papeles, relaciones de poder-dependencia y sexualización de relaciones, factores biográficos que tienen que ver con la situación de la vida personal, víctima y agresor, que en algunas circunstancias pueden favorecer el abuso sexual, lo cual tienen gran relevancia al momento de caracterizar el abuso sexual.

Así mismo es importante identificar y precisar las características físicas del abuso sexual infantil, lo cual (Suarez, 1996) lo define como: "Toda forma de agresión infligida al menor, por sus padres, responsables, o adultos, producida por el uso la fuerza física no accidental. Sus consecuencias pueden ser transitorias o permanentes, incluyendo la muerte. $\mathrm{Su}$ gravedad y época de ocurrencia se gradúa de acuerdo a lo establecido por medicina legal: Leve, moderado, o grave y antigua, reciente o recurrente."

Dentro de las características físicas del abuso sexual que experimentan los niños y niñas se hallan molestias para caminar o sentarse, así como sensaciones de dolor, comezón en el área genital además de visibilizarse está área hinchada, también se pueden llegar a observar manchas de sangre o flujo en la ropa interior, al igual que dolor al orinar, o infecciones urinarias repetitivas, cuerpos extraños en la vagina o en el recto y en algunos casos contusiones o sangrado en las aéreas genitales externas, vaginal o anal. En caso más graves enfermedades de transmisión sexual, VIH positivo y Embarazos prematuros. Cabe señalar que en algunos casos de abuso sexual no siempre se evidencian signos físicos, por lo cual no se debe desestimar otros factores como el relato del niño y las características psicológicas que son importantes a la hora de diagnosticarlo.

Por otro lado la clasificación internacional de enfermedades mentales en su décima edición (Organización Panamericana de la Salud, 1997) CIE 10 incluye al abuso sexual como una crueldad mental; en el que se describen procesos frecuentemente asociados 
con alteraciones mentales y del comportamiento (Pereda N. , 2009). En un estudio donde se hizo una revisión de los principales indicadores psicológicos y sus afectaciones también psicológicas tanto a nivel nacional como internacional desarrollado con víctimas de abuso sexual infantil, encontraron y categorizaron cinco aspectos.

El primer síntoma se denomina Problemas Emocionales: Miedos, fobias, síntomas depresivos, ansiedad, baja autoestima, sentimiento de culpa, estigmatización, trastorno por estrés postraumático, autolesiones, ideación y conducta suicida. Teniendo en cuenta las características que rodean el abuso sexual y las características de la víctima, el impacto emocional varia de una persona a otra dependiendo de la relación abusado- abusador, sobre todo el grado de intimidad establecido entre ellos. La gravedad de las secuelas emocionales está determinada por la forma y estrategias utilizadas por el victimario en función de la frecuencia y duración del abuso sexual (Echeburúa, 2006).

Segundo Problemas Cognitivos: Conducta hiperactiva, problemas atencionales y de concentración, bajo rendimiento académico, funcionamiento cognitivo general no adecuado, trastorno por déficit de atención con hiperactividad. Los niños y niñas abusados sexualmente, pueden presentar diferentes dificultades cognitivas, considerándose uno de los indicadores más notable en el ámbito escolar, produciendo en las victimas un bajo rendimiento académico relacionados con las funciones ejecutivas y en algunos casos trastornos por déficit de atención con hiperactividad.

Tercero Problemas de Relación: Problemas de relación social, caracterizándose por no tener muchos amigos, escaso tiempo de juego con iguales y aislamiento social. En cuanto a la interacción social manifestada en las victimas, se considera una de las mayores dificultades que está presente tanto al inicio como a largo plazo de la experiencia de abuso sexual, creando en estas dificultades en su desarrollo psicosocial.

Cuarto Problemas Funcionales: Problemas de sueño pesadillas, pérdida del control de esfínter enuresis y encopresis, trastorno de la conducta alimentaria, y quejas somáticas. Estos problemas funcionales se evidencian en las enfermedades físicas sin razones médicas, además de las disfuncionalidades del sueño, que tienen relación con las pesadillas asociadas al evento abusivo, por otra parte se ha demostrado en investigaciones recientes la relación de la anorexia y la bulimia con el abuso sexual. (Losada A. S., 2013)

Quinto Problemas de conducta: estos son de tipo compulsivo, disruptivo y disocial, además de tener comportamientos hostiles, agresivos, manifestaciones de ira y rabia, y algunos pueden llegar a presentar el trastorno oposicionista desafiante. También se encuentran conducta sexualizadas como lo son la masturbación de forma compulsiva, la imitación de actos sexuales, la utilización de un vocabulario sexual inapropiado, curiosidad sexual excesiva y conductas exhibicionistas. Browning y Lauman (2001) citado (Pereda, 2010) defienden que la sexualidad desadaptativa es "la consecuencia más extendida del abuso sexual infantil, no obstante, destacan la no existencia de una relación causal entre la experiencia de abuso sexual infantil y el desarrollo de este problema, si bien el abuso sexual infantil actuaría como un importante factor de riesgo a tener en cuenta".

\section{METODOLOGÍA}

El modelo metodológico utilizado fue cuantitativo de tipo descriptivo de corte transversal, para describir las características personales, físicas, psicológicas y ambientales; a partir del análisis de 139 historias clínicas registrado por abuso sexual en una institución del Departamento de Sucre desde los años 2011 a 2014, utilizando el consentimiento informado de la institución para acceder a la información. Igualmente se utilizó una GUIA DE ANALISIS de la historia clínica diseñada por los investigadores y validada por expertos de las ciencias sociales y humanas; entre ellos, dos psicólogos y un Trabajador social. Se pidió el aval institucional para la aplicación de la Guía organizada, asignando un código a cada historia clínica, en los que se consignaron los datos generales del abusado, abusador, ambiente, tipo de abuso sexual, indicadores físicos e indicadores psicológicos y su frecuencia.

A continuación se relaciona la guía utilizada para el análisis de las historias clínicas 


\begin{tabular}{|c|c|c|c|}
\hline $\begin{array}{l}\text { UNIDAD DE } \\
\text { ANÁLISIS }\end{array}$ & VARIABLES & GRADO & OBSERVACIONES \\
\hline \multirow{3}{*}{$\begin{array}{l}\text { 1. Datos Generales } \\
\text { 1.1 Abusado }\end{array}$} & 1. Edad & \begin{tabular}{|l} 
1. 5-9 años \\
2. 10-14 años \\
3. No registra
\end{tabular} & \\
\hline & 2. Sexo & $\begin{array}{l}\text { 1. Masculino } \\
\text { 2. Femenino }\end{array}$ & \\
\hline & 3. Lugar de origen & \begin{tabular}{|l|} 
1. Zona urbana \\
2. Zona rural \\
3. No registra
\end{tabular} & \\
\hline \multirow{4}{*}{ 1.2 Abusador } & 1. Edad & $\begin{array}{l}\text { 1. Mayor de edad } \\
\text { 2. Menor de edad } \\
\text { 3. No registra }\end{array}$ & \\
\hline & 2. Sexo & $\begin{array}{l}\text { 1. Masculino } \\
\text { 2. Femenino }\end{array}$ & \\
\hline & 3. Lugar de origen & \begin{tabular}{|l|} 
1. Zona urbana \\
2. Zona rural \\
3. No registra
\end{tabular} & \\
\hline & 4. Estrategia usada en el abuso & $\begin{array}{l}\text { 1. Soborno } \\
\text { 2. Secuestro } \\
\text { 3. Engaño } \\
\text { 4. Fuerza física } \\
\text { 5. No registra }\end{array}$ & \\
\hline \multirow[t]{2}{*}{ 2. Ambiente } & 1. Lugar del abuso & $\begin{array}{l}\text { 1. Casa } \\
\text { 2.Espacio abierto } \\
\text { 3. Centro escolar } \\
\text { 4. Otros }\end{array}$ & ¿Cuál? \\
\hline & 2. Filiación & $\begin{array}{l}\text { 1. Familiar } \\
\text { 2. Figura de autoridad } \\
\text { 3. Compañero } \\
\text { 4. Amigo } \\
\text { 5. Otros }\end{array}$ & ¿Cuál? \\
\hline 3. Abuso Sexual & Tipo de Abuso & $\begin{array}{l}\text { 1. Con contacto } \\
\text { 2. Sin contacto }\end{array}$ & \\
\hline \multirow{4}{*}{ 4. Indicadores Físicos } & 1- Dificultad para caminar o sentarse & $\begin{array}{l}\text { 1. Presencia } \\
\text { 2. Ausencia } \\
\text { 3. No registra }\end{array}$ & \\
\hline & 2- Dolor, hinchazón o comezón en área genital. & $\begin{array}{l}\text { 1. Presencia } \\
\text { 2. Ausencia } \\
\text { 3. No registra }\end{array}$ & \\
\hline & 3- Manchas de sangre o flujo en la ropa interior & $\begin{array}{l}\text { 1. Presencia } \\
\text { 2. Ausencia } \\
\text { 3. No registra }\end{array}$ & \\
\hline & $\begin{array}{l}\text { 4- Contunciones o sangrado en las áreas genitales } \\
\text { externas, vaginal o anal. }\end{array}$ & $\begin{array}{l}\text { 1. Presencia } \\
\text { 2. Ausencia } \\
\text { 3. No registra }\end{array}$ & \\
\hline
\end{tabular}




\begin{tabular}{|c|c|c|c|}
\hline & 5. Enfermedades transmisión sexual y VIH positivo & $\begin{array}{l}\text { 1. Presencia } \\
\text { 2. Ausencia } \\
\text { 3. No registra }\end{array}$ & \\
\hline & 6. Dolor al orinar o infecciones urinarias repetitivas & $\begin{array}{l}\text { 1. Presencia } \\
\text { 2. Ausencia } \\
\text { 3. No registra }\end{array}$ & \\
\hline & 7. Cuerpos extraños en la vagina o en el recto & $\begin{array}{l}\text { 1. Presencia } \\
\text { 2. Ausencia } \\
\text { 3. No registra }\end{array}$ & \\
\hline & 8. Embarazo prematuro & $\begin{array}{l}\text { 1. Presencia } \\
\text { 2. Ausencia } \\
\text { 3. No registra }\end{array}$ & \\
\hline \multirow{9}{*}{$\begin{array}{l}\text { 5. Indicadores } \\
\text { Psicológicas } \\
\text { 5.1 Conductuales }\end{array}$} & 1. Agresividad & $\begin{array}{l}\text { 1. Una vez } \\
\text { 2. Dos veces } \\
\text { 3. Sistemática } \\
\text { 4. No registra }\end{array}$ & \\
\hline & 2. Hostilidad, ira y rabia & $\begin{array}{l}\text { 1. Presencia } \\
\text { 2. Ausencia } \\
\text { 3. No registra }\end{array}$ & ¿Cuál? \\
\hline & 3. Conformidad compulsiva & $\begin{array}{l}\text { 1. Presencia } \\
\text { 2. Ausencia } \\
\text { 3. No registra }\end{array}$ & \\
\hline & 4. Conducta sexualizada & $\begin{array}{l}\text { 1. Presencia } \\
\text { 2. Ausencia } \\
\text { 3. No registra }\end{array}$ & \\
\hline & 5. Masturbación compulsiva & $\begin{array}{l}\text { 1. Presencia } \\
\text { 2. Ausencia } \\
\text { 3. No registra }\end{array}$ & \\
\hline & 6. Uso de vocabulario sexual inapropiado & $\begin{array}{l}\text { 1. Presencia } \\
\text { 2. Ausencia } \\
\text { 3. No registra }\end{array}$ & \\
\hline & 7. Curiosidad sexual excesiva & $\begin{array}{l}\text { 1. Presencia } \\
\text { 2. Ausencia } \\
\text { 3. No registra } \\
\end{array}$ & \\
\hline & 8. Conductas exhibicionistas & $\begin{array}{l}\text { 1. Presencia } \\
\text { 2. Ausencia } \\
\text { 3. No registra }\end{array}$ & \\
\hline & 9. Imitación de actos sexuales & $\begin{array}{l}\text { 1. Presencia } \\
\text { 2. Ausencia } \\
\text { 3. No registra }\end{array}$ & \\
\hline \multirow{3}{*}{ 5.2 Emocionales } & 1. Fobia & $\begin{array}{l}\text { 1. Presencia } \\
\text { 2. Ausencia } \\
\text { 3. No registra }\end{array}$ & \\
\hline & 2. Síntomas depresivos & $\begin{array}{l}\text { 1. Presencia } \\
\text { 2. Ausencia } \\
\text { 3. No registra }\end{array}$ & \\
\hline & 3. Ansiedad & $\begin{array}{l}\text { 1. Presencia } \\
\text { 2. Ausencia } \\
\text { 3. No registra }\end{array}$ & \\
\hline
\end{tabular}




\begin{tabular}{|c|c|c|}
\hline & 4. Miedo & $\begin{array}{l}\text { 1. Presencia } \\
\text { 2. Ausencia } \\
\text { 3. No registra }\end{array}$ \\
\hline & 5. Baja autoestima & $\begin{array}{l}\text { 1. Presencia } \\
\text { 2. Ausencia } \\
\text { 3. No registra }\end{array}$ \\
\hline & 6. Sentimiento de culpa & $\begin{array}{l}\text { 1. Presencia } \\
\text { 2. Ausencia } \\
\text { 3. No registra }\end{array}$ \\
\hline & 7. Estigmatización & $\begin{array}{l}\text { 1. Presencia } \\
\text { 2. Ausencia } \\
\text { 3. No registra }\end{array}$ \\
\hline & 8. Ideación suicida & $\begin{array}{l}\text { 1. Presencia } \\
\text { 2. Ausencia } \\
\text { 3. No registra }\end{array}$ \\
\hline & 9. Autolesiones & $\begin{array}{l}\text { 1. Presencia } \\
\text { 2. Ausencia } \\
\text { 3. No registra }\end{array}$ \\
\hline & 10. Trastorno por estrés postrauma & $\begin{array}{l}\text { 1. Presencia } \\
\text { 2. Ausencia } \\
\text { 3. No registra }\end{array}$ \\
\hline & 1. Atención y concentración & $\begin{array}{l}\text { 1. Presencia } \\
\text { 2. Ausencia } \\
\text { 3. No registra }\end{array}$ \\
\hline 5.3 Cognitivo & 2. Bajo rendimiento & $\begin{array}{l}\text { 1. Presencia } \\
\text { 2. Ausencia } \\
\text { 3. No registra }\end{array}$ \\
\hline & 3. Trastorno de déficit de atención con hiperactividad & $\begin{array}{l}\text { 1. Presencia } \\
\text { 2. Ausencia } \\
\text { 3. No registra }\end{array}$ \\
\hline & 1. Problemas de relaciones sociales & $\begin{array}{l}\text { 1. Presencia } \\
\text { 2. Ausencia } \\
\text { 3. No registra }\end{array}$ \\
\hline 5.4 Problemas de & 2. menor cantidad de amigos & $\begin{array}{l}\text { 1. Presencia } \\
\text { 2. Ausencia } \\
\text { 3. No registra }\end{array}$ \\
\hline Relaciones & 3. Menor tiempo de juego con iguales & $\begin{array}{l}\text { 1. Presencia } \\
\text { 2. Ausencia } \\
\text { 3. No registra }\end{array}$ \\
\hline & 4. Elevado aislamiento social & $\begin{array}{l}\text { 1. Presencia } \\
\text { 2. Ausencia } \\
\text { 3. No registra }\end{array}$ \\
\hline & 1. Problemas de sueño & $\begin{array}{l}\text { 1. Presencia } \\
\text { 2. Ausencia } \\
\text { 3. No registra }\end{array}$ \\
\hline 5.5 Funciones físicas & 2. Pérdida de control de esfínteres & $\begin{array}{l}\text { 1. Presencia } \\
\text { 2. Ausencia } \\
\text { 3. No registra }\end{array}$ \\
\hline
\end{tabular}




\begin{tabular}{|l|l|l|l|}
\hline \multirow{2}{*}{3. Trastorno de conducta alimentaria } & $\begin{array}{l}\text { 1. Presencia } \\
2 . \text { Ausencia } \\
\text { 3. No registra }\end{array}$ & \\
\cline { 2 - 4 } & \multirow{2}{*}{4. Quejas Somáticas } & $\begin{array}{l}\text { 1. Presencia } \\
\text { 2. Ausencia } \\
\text { 3. No registra }\end{array}$ & \\
& & \\
\hline
\end{tabular}

\section{RESULTADOS}

Tabla 1

Población Víctima de Abuso

\begin{tabular}{cc}
\hline Edad & Porcentaje \\
\hline 6 a 9 años & $46 \%$ \\
10 a 14 años & $54 \%$ \\
\hline
\end{tabular}

En lo que respeta a los datos personales, se clasificaron en dos categorías, abusado y abusador, teniendo en cuenta los rangos poblacionales establecidos y definidos en ésta investigación. En primera instancia, se resalta que el mayor porcentaje (54\%) de la población víctima de abuso sexual sus edades oscilan entre los diez y catorce años, lo que coincide con el desarrollo de caracteres sexuales secundarios, la entrada a la pre adolescencia; sin embargo, es notorio que un $46 \%$. de las víctimas de abuso presentan edades entre los seis a nueve años.

Tabla 2

Género Víctima de Abuso

\begin{tabular}{cc}
\hline Género & Porcentaje \\
\hline Femenino & $51 \%$ \\
Masculino & $49 \%$ \\
\hline
\end{tabular}

En la población de estudio se evidencia que ambos sexos se hayan expuestos al abuso sexual, siendo así que en el género masculinos se presenta en un $49 \%$ y femeninos $51 \%$.
Tabla 3

Zona de residencia de Víctimas de Abuso Sexual

\begin{tabular}{cc}
\hline Zona de Residencia & Porcentaje \\
\hline Urbana & $90 \%$ \\
Rural & $5,1 \%$ \\
No registra & $4,9 \%$ \\
\hline
\end{tabular}

El $90 \%$ de los casos estudiados viven en zona urbana y el $5.1 \%$ viven en zona rural. Resulta alarmante cuando se indaga sobre el lugar de procedencia de las víctimas de abuso sexual, la mayoría presenta un origen urbano, y son escasos los casos cuyos protagonistas procedan de zonas rurales, lo que genera inquietud, puesto que no se sabe con certeza si se debe a la falta de cultura de denuncia, o desconocimiento de canales para realizar las denuncias, intimidación, o es realmente un hecho probado en ésta investigación, que mayor porcentaje de los abusados tienen su lugar de residencia en las zonas urbanas del Departamento de Sucre.

Tabla 4

Edad del abusador

\begin{tabular}{lr}
\hline Edad & Porcentaje \\
\hline 18 años o mas & $54 \%$ \\
Menos de 18 años y 5 años mayores que la victima & $26 \%$ \\
\hline
\end{tabular}

Ahora bien con respecto al abusador, se confirma que son los mayores de edad quienes registran el mayor índice de abusos sobre los menores de edad (54\%); sin embargo el $26 \%$ de los abusadores son menores de edad (5 años mayor que la víctima), es de anotar como la violencia sexual entre pares presenta un porcentaje relevante. 
Tabla 5

Género del Abusador

\begin{tabular}{cc}
\hline Género & Porcentaje \\
\hline Masculino & $97 \%$ \\
Femenino & $3 \%$ \\
\hline
\end{tabular}

El $97 \%$ de los abusadores pertenecen al sexo masculino y el 3\% al femenino. Encontrándose en las historias clínicas estudiadas, que las personas de sexo masculinos presentan mayor tendencia a abusar a otros núcleos poblacionales. En la población estudiada, el $72 \%$ se confirma la tendencia que el origen del abusador es de zonas urbanas, confirmándose la preocupación del por qué en las zonas rurales no se presenta o no se denuncia a los abusadores sexuales.

Tabla 6

Tipos de abuso

\begin{tabular}{cc}
\hline Tipo & Porcentaje \\
\hline Con Contacto & $61 \%$ \\
Sin Contacto & $38 \%$ \\
\hline
\end{tabular}

En cuanto a los tipos de abuso, se encontró que el $61 \%$ de los casos estudiados se realizaron por contacto, el cual involucra un contacto físico con la boca, pechos, genitales, ano o cualquier otra parte del cuerpo del niño. El $38 \%$ sin contacto, es decir no implican contacto físico con el cuerpo del niño, pero si golpean en la salud mental de la víctima.

Tabla 7

Lugar del Abuso

\begin{tabular}{lc}
\hline Lugar & Porcentaje \\
\hline Casa o Hogar & $51 \%$ \\
Espacios Abiertos & $36 \%$ \\
Centros Escolares & $8 \%$ \\
Otros & $6 \%$ \\
\hline
\end{tabular}

Por otra parte, se encontró que el $51 \%$ de los casos estudiados se presentaron en la casa, lo que comprueba que el abusador es alguien de confianza del niño o alguien al cual respeta, quiere y por lo cual el o ella accede. El $36 \%$ se realizaron en espacios abiertos, el $8 \%$ en centros escolares y otros el $6 \%$.
Tabla 8

Estrategias del Abusador

\begin{tabular}{lc}
\hline Estrategias & Porcentaje \\
\hline Soborno & $31 \%$ \\
Engaño & $26 \%$ \\
Fuerza Física & $13 \%$ \\
No registra & $31 \%$
\end{tabular}

También se encontró, que el $31 \%$ utilizaron la estrategia del soborno, el $26 \%$ por engaño, el $13 \%$ la fuerza física y el $31 \%$ no registra. Generalmente el abuso sexual se realiza sometiendo al menor a la fuerza, mediante engaños o sobornos, chantaje emocional o aprovechándose de la relación de mando o poder que se tiene sobre él.

Tabla 9

Filiación

\begin{tabular}{lc}
\hline Filiación & Porcentaje \\
\hline Miembro de familia & $48,7 \%$ \\
Figura de autoridad & $10,3 \%$ \\
Amigo & $18 \%$ \\
Compañero & $10,2 \%$ \\
No registra & $12,8 \%$ \\
\hline
\end{tabular}

De acuerdo a los hallazgos de filiación se encontró que el $48.7 \%$ el abusador es alguien de la familia, lo que confunde más a la víctima, aumentado el sentimiento de culpa, el $10.3 \%$ es una figura de autoridad al cual el niño se somete a satisfacer los deseos sexuales de esté por su indefensión y obediencia, el $18 \%$ es un amigo, el $10.2 \%$ es un compañero y el $12.8 \%$ no registra. Por lo general el victimario antes de abusar sexualmente de un niño o niña gana la confianza de estos para satisfacer sus pretensiones sexuales.

Tabla 10

Indicadores Físicos

\begin{tabular}{lc}
\hline Indicadores & Porcentaje \\
\hline Dificultades para caminar & $5 \%$ \\
Dolor e Hinchazón & $3 \%$ \\
Manchas o Sangre & $6 \%$ \\
Dolor al orinar o infecciones urinarias & $8 \%$ \\
No registra cuerpos extraños en la vagina & $100 \%$ \\
\hline
\end{tabular}


Siguiendo con los indicadores físicos de los casos reportados, el $5 \%$ presenta dificultad para caminar, el 3\% dolor e hinchazón, el $6 \%$ manchas de sangre, el $8 \%$ dolor al orinar o infecciones urinarias, el $100 \%$ no registran cuerpos extraños en la vagina, embarazos no deseados, enfermedades de transmisión sexual, y contusiones o sangrado en las áreas genitales externas, vaginal o anal. Según Suarez (1996), este tipo de abuso infringe al niño cuando es sometido sexualmente por un familiar o adulto ya sea conocido o desconocido. Señala que en algunos casos de abuso sexual no siempre se evidencian signos físicos, por lo cual no se debe desestimar otros factores como el relato del niño y las características psicológicas que son importantes a la hora de diagnosticarlo.

Tabla 11

Indicadores Psicológicos

\begin{tabular}{lc}
\hline \multicolumn{1}{c}{ Indicadores } & Porcentaje \\
\hline Baja autoestima & $51 \%$ \\
Síntomas depresivos & $38 \%$ \\
Ansiedad & $64 \%$ \\
Miedo & $46 \%$ \\
Sentimientos de culpa & $46 \%$ \\
Ideación Suicida & $8 \%$ \\
Trastorno de estrés postraumático & $5 \%$ \\
Fobia & $3 \%$ \\
\hline
\end{tabular}

A sí mismo los indicadores psicológicos, según la clasificación de Pereda 2009, quien plantea que estas se revelan desde lo emocional, cognitivo, problemas funcionales, problemas de relaciones y conductuales. Siendo así la dimensión emocional, confirma la tendencia que las personas que sufren abuso sexual, presentan problemas emocionales severos tales como baja autoestima $51 \%$; síntomas depresivos $38 \%$; ansiedad $64 \%$, miedo $46 \%$; sentimiento de culpa $46 \%$, ideación suicida $8 \%$, trastorno por estrés postraumático $5 \%$, fobia $3 \%$ y estos son en consecuencia, uno de puntos clave para evidenciar el abuso sexual en niños y niñas del departamento de sucre.
Tabla 12

Particularidades cognitivas

\begin{tabular}{lr}
\hline Indicadores & Porcentaje \\
\hline Sin alteración en la atención y concentración & $20,5 \%$ \\
Problemas de atención & $7,7 \%$ \\
Bajo rendimiento académico & $20,5 \%$ \\
Sin bajo rendimiento académico & $5,1 \%$ \\
Trastorno de déficit de atención con hiperactividad & $2,6 \%$
\end{tabular}

En relación a las particularidades cognitivas, se resalta que el $20.5 \%$ presentan atención y concentración, el $7.7 \%$ tienen problemas de atención, el $20.5 \%$ bajo rendimiento, el $5.1 \%$ no tienen bajo rendimiento, el $2.6 \%$ trastorno de déficit de atención con hiperactividad, características que son clasificadas por Pereda 2009 con Conducta hiperactivas, problemas de atención y concentración, bajo rendimiento académico, peor funcionamiento cognitivo general, trastorno por déficit de atención con hiperactividad.

Tabla 13

Problemas Funcionales

\begin{tabular}{lc}
\hline \multicolumn{1}{c}{ Indicadores } & Porcentaje \\
\hline Alteración del Sueño & $31 \%$ \\
Trastorno Alimenticio & $8 \%$ \\
Quejas somáticas & $5 \%$ \\
\hline
\end{tabular}

Por otra parte los problemas funcionales que más prevalecen son los relacionados con el sueño y se presenta en un $31 \%$, seguido en menor porcentaje por los trastornos alimenticios en un $8 \%$ y quejas somáticas en un $5 \%$, lo cual no deja de preocupar, dado que las consecuencias de estas complicaciones pueden generar resultados nefastos en la salud de las víctimas.

Tabla 14

Problemas Relacionales

\begin{tabular}{lc}
\hline Indicadores & Porcentaje \\
\hline Problemas en las relaciones sociales & $10 \%$ \\
Menor cantidad de amigos & $23 \%$ \\
Presentan amigos & $8 \%$ \\
No tienen tiempo de juego con los pares & $18 \%$ \\
Tiempo de juego con pares & $2 \%$ \\
Aislamiento social & $18 \%$ \\
Sin aislamiento social & $5 \%$ \\
\hline
\end{tabular}


Además en correspondencia con los problemas de relaciones el $10 \%$ presentan problemas de relaciones sociales, el 23\% menor cantidad de amigos, el $8 \%$ presentan amigos, el $18 \%$ no tienen tiempo de juego con los pares y el $2 \%$ tienen tiempo de juegos de pares, el $18 \%$ elevado aislamiento social y el $5 \%$ no presentan aislamiento social. Estas características contrastan con la teoría de Pereda 2009, quien las clasifica en Problemas de relación social, menor cantidad de amigos, menor tiempo de juego con iguales, elevado aislamiento social.

Tabla 15

Problemas Relacionales

\begin{tabular}{lc}
\hline \multicolumn{1}{c}{ Indicadores } & Porcentaje \\
\hline Hostilidad, ira y rabia & $38 \%$ \\
Conformidad compulsiva & $5 \%$ \\
Conducta sexualizada & $15,4 \%$ \\
Vocabulario sexual inapropiado & $10 \%$ \\
Curiosidad & $5 \%$ \\
Conductas exhibicionista & $10 \%$ \\
Conductas de actos sexuales & $13 \%$ \\
\hline
\end{tabular}

Así mismo según el estudio realizado las características conductuales, como la hostilidad, ira y rabia se presenta en un $38 \%$, el $5 \%$ presencia de conformidad compulsiva, el $15 \%$ conducta sexualidad, el $15.4 \%$ presencia de masturbación compulsiva,el $10 \%$ presentan un vocabulario sexual inapropiado, el 5\% presentan curiosidad, el $10 \%$ conductas exhibicionista y el 13\% imitan conductas de actos sexuales. Resultados que contrastan con la teoría que plantea que las secuelas conductuales de un abuso sexual al menor presenta; Conducta sexualidad: masturbación compulsiva, imitación de actos sexuales, uso de vocabulario sexual inapropiado, curiosidad sexual excesiva y conductas exhibicionistas. Conformidad compulsiva. Conducta disruptiva y disocial Hostilidad, agresividad, ira y rabia, Trastorno oposicionista desafiante, Pereda (2009).

\section{DISCUSIÓN}

Los niños de que tienen mayor riesgo de ser abusado sexualmente se encuentran en edades de 10 a 12 años, aunque el porcentaje de diferencia con los niños de 6 a 9 es poca, es importante denotar que en cuanto al sexo el femenino presenta mayor incidencia de abuso, aunque el sexo masculino no está excepto de ser abusado, ni tampoco dista mucho del porcentaje de niñas abusadas, lo cual indica que ambos sexos están expuestos a este tipo de situación, (Cantón D y Justicia F, 2008). En ese sentido son los adultos los que en su mayoría causan este tipo de maltrato, empleado para el logro de su objetivo el soborno y el chantaje como su principal estrategia, cabe resaltar también que esta situación se presenta entre iguales en menor grado, situación que pone en alerta a los equipos psicológicos y juzgados de familia (Horno, Santos y Del Molino, 2001). Por otro lado los infantes de las zonas urbanas son los más vulnerables, tal vez porque hay mayor cercanía del lugar de origen de los abusadores y sus víctimas. Siendo el sexo masculino los que más abusan sexualmente de niños y niñas, aunque las mujeres también incurren en el hecho pero en menor grado, esto quiere decir que tanto hombres como mujeres, pueden incurrir en este tipo de maltrato.

Los casos de mayor frecuencia se reportan en la casa, y en segundo lugar otros lugares, siendo los agresores las personas más cercanas a los infantes, estos pueden ser un familiar o un conocido y en otros casos desconocidos. De acuerdo con Faller (1993) existen ciertas características del contexto como las mencionadas anteriormente que pueden ser tenidas en cuenta al momento de describir las situaciones ambientales, sociales y personales tanto del abusado como del abusador, que pueden favorecer el abuso sexual, mas no lo provocan.

A su vez con respecto al tipo de abuso más reportado es el que se da con contacto en donde existe algún tocamiento o penetración, presentando algunas características físicas como dificultad para caminar, dolor e hinchazón, manchas de sangre y contusiones. Algunos indicadores muestran que el tipo sin contacto también se presenta, pero es más difícil demostrarlo por no tener evidencias, dado que la veracidad de los hechos es puesta en duda.

Por otro lado se evidencian en los resultados la magnitud de la afección psicológica y social que deja el abuso sexual en infantes y adolescentes, tanto en sus inicios como a largo plazo (Pereda, 2010) comprometiendo seriamente sus esferas psíquicas, las 
cuales deja secuelas a lo largo de la vida, interfiriendo en el desarrollo de su personalidad (Pereda G. J., 2011), claro está teniendo en cuenta el tipo de abuso al que fue sometido, la edad en que ocurrió el hecho, si es a nivel intrafamiliar o extrafamiliar (Ysern De Arce, 2006) de acuerdo al vínculo establecido entre el abusado y abusador. En este sentido las investigaciones demuestran que los casos de abuso sexual más frecuentes son las realizadas por personas cercanas al infante, ganándose su confianza y aprovechándose de su figura de autoridad (Intebi, 1998) y así someterlos a sus pretensiones sexuales creando en este inseguridades a nivel emocional, cognitivo, funcional, comportamental y social (Pereda, 2010).

Cabe resaltar, que los niños que han sido abusados de forma continua y por un largo tiempo pueden desarrollar una pérdida de la autoestima, menosprecian su valor y adquieren un concepto erróneo de la sexualidad. Pueden volverse retraídos y desconfiados, llegando a considerar el suicidio (Pereda, 2010). Los primeros indicadores de un abuso sexual pueden ser manifestaciones como aplanamiento afectivo, irritabilidad, hipervigilancia, ansiedad, sentimientos de culpa, vergüenza, daño en la propia imagen, en la parte físico genital y el usual contagio de ITS. Posterior a estos indicadores, se suman reacciones de forma inmediata o tardías como la depresión, problemas o trastornos de conducta, mal rendimiento escolar, consumo de sustancias, hipersexualidad, disfunción sexual, intentos suicidas, somatizaciones, trastornos de personalidad, entre otras.

El reconocimiento del abuso sexual a nivel social y profesional ha permitido un incremento en los casos que se conocen, aunque solo represente un porcentaje mínimo a la incidencia real. Además, el abuso sexual en niños y niñas resulta un tema de investigación difícil y problemático que incluye el secreto, la vergüenza y la culpa hacia la persona afectada, sacando a la luz tabúes que comprometen la sexualidad, el poder, los valores y actitudes hacia la infancia.

Finalmente atendiendo a todas estas problemáticas, que atentan en contra de la salud física y psicológica de niños, niñas y adolescentes de todas las esferas a nivel mundial las Naciones Unidas ha declarado en el informe de los derechos del niño (ONU, 2008) que "ningún niño sea sometido a torturas ni a otros tratos o penas crueles, inhumanos o degradantes".

Esta afirmación se complementa y amplía en el artículo 19, que estipula que los "Estados adoptarán todas las medias legislativas, administrativas, sociales y educativas apropiadas para proteger al niño contra toda forma de perjuicio o abuso físico o mental, descuido o trato negligente, malos tratos o explotación, incluido el abuso sexual, mientras el niño se encuentre bajo la custodia de los padres, de un representante legal o de cualquier otra persona que lo tenga a su cargo". No hay ninguna ambigüedad: la expresión "toda forma de perjuicio o abuso físico o mental" no deja espacio para ningún grado de violencia legalizada contra los niños. Los castigos corporales y otras formas de castigo cruel o degradante son formas de violencia y perjuicio ante las que los Estados deben adoptar todas las medidas legislativas, administrativas, sociales y educativas apropiadas para eliminarlas".

Así mismo Colombia protege a los niños, niñas y adolescentes por medio Código de la Infancia y la Adolescencia, y el Código de Procedimiento Penal de la Constitución política 1991 (Castro, 2008) ante toda forma de abandono, de violencia física o moral, de secuestro o venta, de abuso sexual, de explotación económica y laboral, y de los trabajos riesgosos. Agrega que gozarán de los derechos consagrados en la Constitución, en las leyes y en los tratados internacionales ratificados (Art. 44).

\section{REFERENCIAS}

Alonso, J., \& Horno, P. (2004). Una experiencia de buena práctica en intervención sobre el abuso sexual infantil: Advocancy: construcción de redes y formación. España: Save the children.

Cantón D, \&., Justicia ,F. (2008). Afrontamiento del abuso sexual infantil y ajuste psicológico a largo plazo. Psicothema, 509- 515.

Cantón, D., \& Cortés, M. (mayo de 2015). Consecuencias del abuso sexual infantil: una revisión de las variables intervinientes. Anales de la psicología, 31(2), 552-561.

Castro, J. (2008). Constitucion Politica de Colombia. Bogotá- Colombia: Universidad Del Rosario. 
Cely, L. A. (2003). Intervención interdisciplinaria en casos de abuso sexual infantil. Universitas Psychologica, 57-60.

Children, S. T. (2004). Una experiencia de buena práctica en intervención sobre el abuso sexual infantil: Advocacy: construcción de redes y formación. España.

Echeberúa, P., \& De Corral, P. (2006). Secuelas en victimas de abuso sexual en la infancia. Guad Med Forense, 43-44.

Echeburúa. (2006). Secuelas emocionales en víctimas de abuso. Cuad Med Forense, 75-82.

Echeburúa, G. (2005). Abuso sexual en la infancia: víctimas y agresores: un enfoque clínico. España: Ariel .

Guerricaechevarria, C., \& Echeverúa, E. (2005). Abuso sexual en la infancia: victimas y agresores: un enfoque clinico. España: Ariel.

Horno, P., Santos, A., \& Del Molino, A. (2001). Abuso sexual infantil: Manual de formación para profesionales. España: Save the children.

ICBF. (18 de Septiembre de 2013). Icbf ha recibido 4.710 casos por violencia sexual infantil en 2013 . El Tiempo.

Intebi, I. (1998). Abuso sexual infantil en las mejores familias. Barcelona-España: Granica.

López-Ibor, J.; Valdez Millar, M. (2002). DSM-IV-TR: Manual diagnóstico y estadístico de los trastornos mentales, Texto revisado. 4ta. edición. Barcelona: Masson.

Losada. (2012). Epidemiologia del abuso sexual infantil. GEPU, 201-229.

Losada, A. S. (2013). Abuso sexual infantil, trastornos de la conducta. Psicología, Conocimiento y Sociedad, 102-134.

Losada, C. y. (2009). Nuevos Aportes Al abuso Sexual Infantil. Buenos Aires: R/V Ediciones-Ediciones Kristal.

Fecha de recepción: 3 de junio de 2016

Fecha de aceptación: 12 de junio de 2016
Manso, J. M. (2006). Revisión de los principales modelos teóricos explicativos del maltrato infantil. Enseñanza e Investigación en Psicología, 271-292.

Mebarak, M. S. (2010). Una revisión acerca de la sintomatología del abuso sexual infantil. Psicologia desde el caribe, 128-154.

Medicina Legal. (10 de Octubre de 2013). Cada hora, dos niñas son víctimas de abuso sexual en Colombia. El Pais.com.co.

ONU. (2008). Informe del Comité de Los Derechos del Niño. Nueva York: ONU.

Organización Panamericana de la Salud. (1.997). CIE 10. Washington. Estados Unidos: Organización Panamericana de la Salud.

Pereda. (2010). Consecuencias psicologicas a largo plazo del abuso sexual infantil. Papeles del Psicólogo, 191-201.

Pereda, G. J. (2011). Trastornos de personalidad en víctimas de abuso sexual infantil. Actas españolas de Psiquiatría, 131-139.

Pereda, N. (2009). Consecuencias psicológicas iniciales. Papeles del Psicólogo, 135-144.

Rodrígez, L. (2003). Intervención interdisciplinaria en casos de abuso sexual infantil. Redalyc.org, 5770 .

Save the children. (2001). Abuso sexual infantil: Manual de formacion para profesionales. España: Save the children

Suarez. (1996). Maltrato infantil en Colombia. CES. Medicina, 10 (2).

Ysern De Arce, y. B. (2006). Abuso sexual intrafamiliar: Prevalencia y características en jóvenes de $3^{\circ}$ medio de liceos. Theoria, 79-85.

Ysern, J., \& Becerra, P. (2006). Abuso sexual: prevalencia y caracteristicas en jovenes de $3^{\circ}$ medio de liceos municipalizados de Chillán, Chile. Theoria: Ciencia, arte y humanidades, 79-85. 